\title{
Electroweak phase transition in the economical 3-3-1 model
}

\author{
Vo Quoc Phong ${ }^{1, \mathrm{a}}$, Hoang Ngoc Long ${ }^{2, \mathrm{~b}}$, Vo Thanh Van ${ }^{1, \mathrm{c}}$, Le Hoang Minh ${ }^{1, \mathrm{~d}}$ \\ ${ }^{1}$ Department of Theoretical Physics, Ho Chi Minh City University of Science, Ho Chi Minh City, Vietnam \\ ${ }^{2}$ Institute of Physics, Vietnamese Academy of Science and Technology, 10 Dao Tan, Ba Dinh, Hanoi, Vietnam
}

Received: 8 April 2015 / Accepted: 30 June 2015 / Published online: 22 July 2015

(C) The Author(s) 2015. This article is published with open access at Springerlink.com

\begin{abstract}
We consider the EWPT in the economical 3-3-1 (E331) model. Our analysis shows that the EWPT in the model is a sequence of two first-order phase transitions, $S U(3) \rightarrow S U(2)$ at the $\mathrm{TeV}$ scale and $S U(2) \rightarrow U(1)$ at the $100 \mathrm{GeV}$ scale. The EWPT $S U(3) \rightarrow S U(2)$ is triggered by the new bosons and the exotic quarks; its strength is about $1-13$ if the mass ranges of these new particles are $10^{2}-10^{3} \mathrm{GeV}$. The EWPT $S U(2) \rightarrow U(1)$ is strengthened by only the new bosons; its strength is about $1-1.15$ if the mass parts of $H_{1}^{0}, H_{2}^{ \pm}$and $Y^{ \pm}$are in the ranges $10-10^{2} \mathrm{GeV}$. The contributions of $H_{1}^{0}$ and $H_{2}^{ \pm}$to the strengths of both EWPTs may make them sufficiently strong to provide large deviations from thermal equilibrium and B violation necessary for baryogenesis.
\end{abstract}

\section{Introduction}

In the context of electroweak baryogenesis (EWBG), the EWPT plays an important role in explaining the Baryon Asymmetry of Universe (BAU) by electroweak physics. From the three Sakharov conditions, which are B violation, $\mathrm{C}$ and $\mathrm{CP}$ violations, and deviation from thermal equilibrium [1], the EWPT should be a strongly first-order phase transition. That not only leads to thermal imbalance [2], but it also makes a connection between $\mathrm{B}$ violation and $\mathrm{CP}$ violation via nonequilibrium physics [3,4].

The EWPT has been investigated in the Standard Model (SM) [2,5-8] as well as various extension models [9-23]. For the SM, although the EWPT strength is larger than unity at the electroweak scale, it is still too weak for the mass of the Higgs boson to be compatible with current experimental limits [2,5-8]; this suggests that EWBG requires new physics

\footnotetext{
a e-mail: vo.quoc.phong.vl@gmail.com

be-mail: hnlong@iop.vast.ac.vn

c e-mail: vtvan@hcmus.edu.vn

de-mail:1hminh167@gmail.com
}

beyond the SM at the weak scale [9-11]. Many extensions such as the Two-Higgs-Doublet model or Minimal Supersymmetric Standard Model have a more strongly first-order phase transition and the new sources of $\mathrm{CP}$ violation, which are necessary to account for the BAU; triggers for the firstorder phase transition in these models are heavy bosons or dark matter candidates [15-17,19-23].

Among the extensions beyond the SM, the models based on $S U(3)_{C} \otimes S U(3)_{L} \otimes U(1)_{X}$ gauge group (called 3-3-1 for short) [24-32] have some interesting features including the ability to explain the generation problem [24-32] and the electric charge quantization [33-36]. The structure of such a gauge group requires the 3-3-1 models to have at least two Higgs triplets. Thus the structure of symmetry breaking and the number of bosons are different from those in the SM.

In a previous work [37], we have considered the EWPT in the reduced minimal 3-3-1 (RM331) model due to its simplicity, and found that our approach can be applied to the more complicated 3-3-1 models. In the present work, we follow the same approach for the economical 3-3-1 (E331) model [38-44], whose lepton sector is more complicated than that of the RM331 model. The E331 model has the right-handed neutrino in the leptonic content, the bileptons (two singly charged gauge bosons $W^{ \pm}, Y^{ \pm}$, and a neutral gauge bosons $X^{0}$ ), the heavy neutral boson $Z_{2}$, and the exotic quarks. The model has two Higgs triplets, and the physical scalar spectrum is composed of a singly charged scalar $H_{2}^{ \pm}$and a neutral scalars $H_{1}^{0}$ [38-44]. We will show in this paper that the new bosons and the exotic quarks can be triggers for the first-order phase transition in the model.

This paper is organized as follows. In Sect. 2 we give a review of the E331 model on the Higgs, gauge boson, and lepton sectors. In Sect. 3, we find the effective potential in the model, which has a contribution from heavy bosons and exotic quarks as well as a contribution similar to that in the SM. In Sect. 4, we investigate the structure of the EWPT sequence in the E331 model, we find the parameter ranges where the EWPTs are strongly first-order to provide B viola- 
tion necessary for baryogenesis, and we show the constraints on the mass of the charged Higgs boson. Finally, we summarize and describe outlooks in Sect. 5.

\section{A review of the economical 331 model}

\subsection{Higgs potential}

In the E331 model, the 3-3-1 gauge group is spontaneously broken via two stages. In the first stage, the group $S U(3)_{L} \otimes$ $U(1)_{X}$ breaks down to the $S U(2)_{L} \otimes U(1)_{Y}$ of the SM; and the second stage takes place as that we have known in the SM. This sequence of spontaneous symmetry breaking (SSB) is described by the Higgs potential [38-44]:

$$
\begin{aligned}
V(\chi, \phi)= & \mu_{1}^{2} \chi^{\dagger} \chi+\mu_{2}^{2} \phi^{\dagger} \phi+\lambda_{1}\left(\chi^{\dagger} \chi\right)^{2}+\lambda_{2}\left(\phi^{\dagger} \phi\right)^{2} \\
& +\lambda_{3}\left(\chi^{\dagger} \chi\right)\left(\phi^{\dagger} \phi\right)+\lambda_{4}\left(\chi^{\dagger} \phi\right)\left(\phi^{\dagger} \chi\right),
\end{aligned}
$$

in which $\chi$ and $\phi$ are the Higgs scalar triplets:

$\chi=\left(\begin{array}{l}\chi_{1}^{0} \\ \chi_{2}^{-} \\ \chi_{3}^{0}\end{array}\right) \sim\left(1,3,-\frac{1}{3}\right), \quad \phi=\left(\begin{array}{c}\phi_{1}^{+} \\ \phi_{2}^{0} \\ \phi_{3}^{+}\end{array}\right) \sim\left(1,3,-\frac{1}{3}\right)$,

whose VEVs are, respectively, given by

$$
\langle\chi\rangle=\frac{1}{\sqrt{2}}\left(\begin{array}{c}
u \\
0 \\
\omega
\end{array}\right), \quad\langle\phi\rangle=\frac{1}{\sqrt{2}}\left(\begin{array}{l}
0 \\
v \\
0
\end{array}\right)
$$

where the VEV $\omega$ is responsible for the first stage, and the VEVs $u$ and $v$ are responsible for the second stage of symmetry breaking. These VEVs satisfy the constraint [38-44]:

$\omega \gg v \gg u$.

The physical scalar spectrum of the model is composed of a charged scalar $H_{2}^{+}$, and two neutral scalars $H_{1}^{0}$ and $H^{0}$. In this spectrum, $H^{0}$ is both the lightest neutral field and a $S U(2)_{L}$ component, hence it is identified as the SM Higgs boson. The Higgs content of the model can be summarized as follows:

$$
\begin{gathered}
\chi=\left(\begin{array}{c}
\frac{1}{\sqrt{2}} u+G_{X^{0}} \\
G_{Y^{-}} \\
\frac{1}{\sqrt{2}}\left(\omega+H_{1}^{0}+i G_{Z^{\prime}}\right)
\end{array}\right), \\
\phi=\left(\begin{array}{c}
G_{W^{+}} \\
\frac{1}{\sqrt{2}}\left(v+H^{0}+i G_{Z}\right) \\
H_{2}^{+}
\end{array}\right),
\end{gathered}
$$

where the Higgs masses are given by

$$
\begin{aligned}
m_{H^{0}}^{2}= & \lambda_{2} v^{2}+\lambda_{1}\left(u^{2}+\omega^{2}\right) \\
& -\sqrt{\left[\lambda_{2} v^{2}-\lambda_{1}\left(u^{2}+\omega^{2}\right)\right]^{2}+\lambda_{3}^{2} v^{2}\left(u^{2}+\omega^{2}\right)} \\
\approx & \frac{4 \lambda_{1} \lambda_{2}-\lambda_{3}^{2}}{2 \lambda_{1}} v^{2}, \\
m_{H_{1}^{0}}^{2}= & \lambda_{2} v^{2}+\lambda_{1}\left(u^{2}+\omega^{2}\right) \\
& +\sqrt{\left[\lambda_{2} v^{2}-\lambda_{1}\left(u^{2}+\omega^{2}\right)\right]^{2}+\lambda_{3}^{2} v^{2}\left(u^{2}+\omega^{2}\right)} \\
\approx & 2 \lambda_{1} \omega^{2}+\frac{\lambda_{3}^{2}}{2 \lambda_{1}} v^{2}, \\
m_{H_{2}^{+}}^{2}= & \frac{\lambda_{4}}{2}\left(u^{2}+v^{2}+\omega^{2}\right) .
\end{aligned}
$$

We note that in Ref. [38-44], the mass formula of $H_{1}^{0}$ is approximate as $m_{H_{1}^{0}}^{2} \approx 2 \lambda_{1} \omega^{2}$. In the context of EWPT, however, we find that the better approximation should be that in Eq. (7). Although the additional term $\frac{\lambda_{3}^{2}}{2 \lambda_{1}} v^{2}$ is very small as compared to the first term and we may neglect it in some other considerations, it gives a very important contribution of $H_{1}^{0}$ to the EWPT $S U(2) \rightarrow U(1)$.

\subsection{Gauge boson sector}

The masses of the gauge bosons of this model come from the Lagrangian

$\mathcal{L}_{\text {mass }}^{G B}=\left(\mathcal{D}_{\mu} \chi\right)^{\dagger}\left(\mathcal{D}^{\mu} \chi\right)+\left(\mathcal{D}_{\mu} \phi\right)^{\dagger}\left(\mathcal{D}^{\mu} \phi\right)$,

where

$\mathcal{D}_{\mu}=\partial_{\mu}-i g T_{i} W_{i \mu}-i g_{X} T_{9} X B_{\mu}$,

with $T_{9}=\frac{1}{\sqrt{6}} \operatorname{diag}(1,1,1)$ so that $\operatorname{Tr}\left(T_{i} T_{j}\right)=\delta_{i j}$. The couplings of $S U(3)_{L}$ and $U(1)_{X}$ satisfy the relation

$t \equiv \frac{g_{X}}{g}=\frac{3 \sqrt{2} s_{W}}{3-4 s_{W}^{2}}$,

where $c_{W}=\cos \theta_{W}, s_{W}=\sin \theta_{W}, t_{W}=\tan \theta_{W}$, and $\theta_{W}$ is the Weinberg angle.

Equations (9) and (3) lead to

$W_{\mu}^{\prime \pm}=\frac{W_{1 \mu} \mp i W_{2 \mu}}{\sqrt{2}}, \quad Y_{\mu}^{\prime \mp}=\frac{W_{6 \mu} \mp i W_{7 \mu}}{\sqrt{2}}$,

$m_{W_{5}}^{2}=\frac{g^{2}}{4}\left(v^{2}+\omega^{2}\right)$.

The combinations $W^{\prime}$ and $Y^{\prime}$ in (12) are mixed via a mass matrix:

$\mathcal{L}_{\text {mass }}^{C G}=\frac{g^{2}}{4}\left(W_{\mu}^{\prime-}, Y_{\mu}^{\prime-}\right)\left(\begin{array}{cc}u^{2}+v^{2} & u \omega \\ u \omega & \omega^{2}+v^{2}\end{array}\right)\left(\begin{array}{c}W^{\prime+\mu} \\ Y^{\prime-\mu}\end{array}\right)$. 
Diagonalizing the mass matrix in Eq. (14), we obtain the physical charged gauge bosons

$W_{\mu}=\cos \theta W_{\mu}^{\prime}-\sin \theta Y_{\mu}^{\prime}, \quad Y_{\mu}=\sin \theta W_{\mu}^{\prime}-\cos \theta Y_{\mu}^{\prime}$

and their respective mass eigenvalues

$m_{W}^{2}=\frac{g^{2} v^{2}}{4}, m_{Y}^{2}=\frac{g^{2}}{4}\left(u^{2}+v^{2}+\omega^{2}\right)$,

where $\theta$ is the mixing angle which is defined by

$t_{\theta} \equiv \tan \theta=\frac{u}{\omega}$.

The mass $m_{W}$ as in (16) suggests that the $W$ bosons of the model can be identified as those of the SM, and $v$ can be set as $v \simeq v_{\text {weak }}=246 \mathrm{GeV}$. From the constraints in (4), $\theta$ should be very small, thus $W_{\mu} \simeq W_{\mu}^{\prime}$ and $Y_{\mu} \simeq Y_{\mu}^{\prime}$. Moreover, the Michel parameter $\rho$ in the model connects $u$ with $v$ by the expression $\rho \approx 1+\frac{3 u^{2}}{v^{2}}$ [38-44]; and from the experimental data, $\rho=0.9987 \pm 0.0016$ [45], that expression gives us $\frac{u}{v} \leq 0.01$, which leads to $u<2.46 \mathrm{GeV}$. With $\omega$ in the range $1-5 \mathrm{TeV}$, we have

$t_{\theta}=\frac{u}{\omega} \approx 0.001$.

For the neutral gauge bosons, the mass matrix in the basis $\left(W_{3 \mu}, W_{8 \mu}, B_{\mu}, W_{4 \mu}\right)$ is given by
Due to the constraints (4), the physical states $Z_{1}$ and $Z_{2}$ get masses

$m_{Z_{1}}^{2}=\frac{g^{2}}{4 c_{W}^{2}}\left(v^{2}-3 u^{2}\right), \quad m_{Z_{2}}^{2}=\frac{g^{2} c_{W}^{2} \omega^{2}}{3-4 s_{W}^{2}}$.

Since the components $W_{4}^{\prime}$ and $W_{5}$ have the same mass, we can identify their combination,

$X_{\mu}^{0}=\frac{1}{\sqrt{2}}\left(W_{4 \mu}^{\prime}-i W_{5 \mu}\right)$

as a physical neutral non-Hermitian gauge boson which carries the lepton number with two units. The subscript 0 of $X_{\mu}$ in Eq. (24) denotes the neutrality of the gauge boson $X$, but sometimes this subscript may be dropped.

\subsection{Fermion sector}

The fermion content in this model, which is anomaly free, is given by

$$
\begin{aligned}
\psi_{i L} & =\left(\begin{array}{c}
v_{i} \\
e_{i} \\
\chi_{i}^{0}
\end{array}\right)_{L} \sim\left(1,3,-\frac{1}{3}\right), \\
e_{i R} & \sim(1,1,-1), \quad i=1,2,3, \\
Q_{1 L} & =\left(\begin{array}{l}
u_{1} \\
d_{1} \\
U
\end{array}\right)_{L} \sim\left(3,3, \frac{1}{3}\right),
\end{aligned}
$$

$$
\begin{aligned}
M^{2}= & \frac{g^{2}}{4} \\
& \times\left(\begin{array}{cccc}
u^{2}+v^{2} & \frac{u^{2}-v^{2}}{\sqrt{3}} & -\frac{2 t}{3 \sqrt{6}}\left(u^{2}+2 v^{2}\right) & 2 u \omega \\
\frac{u^{2}-v^{2}}{\sqrt{3}} & \frac{1}{3}\left(4 \omega^{2}+u^{2}+v^{2}\right) & \frac{\sqrt{2} t}{9}\left(2 \omega^{2}-u^{2}+2 v^{2}\right) & -\frac{2}{\sqrt{3}} u \omega \\
-\frac{2 t}{3 \sqrt{6}}\left(u^{2}+2 v^{2}\right) & \frac{2 t}{9}\left(2 \omega^{2}-u^{2}+2 v^{2}\right) & -\frac{2 t^{2}}{27}\left(\omega^{2}+u^{2}+4 v^{2}\right)-\frac{8 t}{3 \sqrt{6}} u \omega \\
2 u \omega & -\frac{2}{\sqrt{3}} u \omega & -\frac{8 t}{3 \sqrt{6}} u \omega & u^{2}+\omega^{2}
\end{array}\right) .
\end{aligned}
$$

The diagonalization of the mass matrix in Eq. (19) leads to the mass eigenstates of four following neutral gauge bosons:

$$
\begin{aligned}
m_{\gamma}^{2}= & 0, \quad m_{W_{4}^{\prime}}^{2}=\frac{g^{2}}{4}\left(u^{2}+\omega^{2}\right), \\
m_{Z_{1}}^{2}= & {\left[2 g^{-2} \sqrt{3-4 s_{W}^{2}}\right]^{-1}\left\{\left[c_{W}^{2}\left(u^{2}+\omega^{2}\right)+v^{2}\right.\right.} \\
& \left.-\sqrt{\left[c_{W}^{2}\left(u^{2}+\omega^{2}\right)+v^{2}\right]^{2}+\left(3-4 s_{W}^{2}\right)\left(3 u^{2} \omega^{2}-u^{2} v^{2}-v^{2} \omega^{2}\right)}\right\},
\end{aligned}
$$

$$
\begin{aligned}
m_{Z_{2}}^{2}= & {\left[2 g^{-2} \sqrt{3-4 s_{W}^{2}}\right]^{-1}\left\{\left[c_{W}^{2}\left(u^{2}+\omega^{2}\right)+v^{2}\right.\right.} \\
& \left.+\sqrt{\left[c_{W}^{2}\left(u^{2}+\omega^{2}\right)+v^{2}\right]^{2}+\left(3-4 s_{W}^{2}\right)\left(3 u^{2} \omega^{2}-u^{2} v^{2}-v^{2} \omega^{2}\right)}\right\} .
\end{aligned}
$$

$$
\begin{gathered}
Q_{\alpha L}=\left(\begin{array}{c}
d_{\alpha} \\
u_{\alpha} \\
D_{\alpha}
\end{array}\right)_{L} \sim\left(3,3^{*}, 0\right), \quad \alpha=2,3, \\
u_{i R} \sim\left(3,1, \frac{2}{3}\right), \quad d_{i R} \sim\left(3,1,-\frac{1}{3}\right), \\
u_{R} \sim\left(3,1, \frac{2}{3}\right), \quad D_{\alpha R} \sim\left(3,1,-\frac{1}{3}\right) .
\end{gathered}
$$

The Yukawa interactions which induce masses for the fermions can be written as

$$
\mathcal{L}_{Y u k}=\mathcal{L}_{L N C}+\mathcal{L}_{L N V}
$$


in which $\mathcal{L}_{L N C}$ is the Lagrangian part for lepton-number conservation and $\mathcal{L}_{L N V}$ is that for lepton-number violation. These Lagrangian parts are given by

$$
\begin{aligned}
& \mathcal{L}_{L N C}=h^{U} \bar{Q}_{1 L \chi} U_{R}+h_{\alpha \beta}^{D} \bar{Q}_{\alpha L} \chi^{*} D_{\beta R} \\
& \quad+h_{i j}^{e} \bar{\psi}_{i L} \phi e_{j R}+h_{i j}^{\epsilon} \epsilon_{a b c}\left(\bar{\psi}_{i L}^{c}\right)_{a}\left(\psi_{j L}^{c}\right)_{b}(\phi)_{c} \\
& \quad+h_{i}^{d} \bar{Q}_{1 L} \phi d_{i R}+h_{\alpha i}^{u} \bar{Q}_{\alpha L} \phi^{*} u_{i R}+H . c . \\
& \mathcal{L}_{L N V}=s_{i}^{u} \bar{Q}_{1 L} \chi u_{i R}+s_{\alpha i}^{d} \bar{Q}_{\alpha L} \chi^{*} d_{i R} \\
& \quad+s_{\alpha}^{D} \bar{Q}_{1 L} \phi D_{\alpha R}+s_{\alpha}^{U} \bar{Q}_{\alpha L} \phi^{*} U_{R}+H . c .
\end{aligned}
$$

where $a, b$, and $c$ stand for the $S U(3)_{L}$ indices.

During the SSB sequence of this model, the VEV $\omega$ gives the masses for the exotic quarks $U$ and $D_{\alpha}$, the VEV $u$ which is the source of lepton-number violations gives the masses for the quarks $u_{1}$ and $d_{\alpha}$, the VEV $v$ gives the masses for the quarks $u_{\alpha}$ and $d_{1}$ as well as all ordinary leptons.

\section{Effective potential in the economical 331 model}

From the Higgs potential (1), we obtain $V_{0}$ in a form which is dependent on the VEVs as follows:

$$
\begin{aligned}
V_{0}(u, \omega, v)= & \frac{\mu_{1}^{2}}{2}\left(u^{2}+\omega^{2}\right)+\frac{\mu_{2}^{2}}{2} v^{2}+\frac{\lambda_{1}}{4}\left(u^{4}+\omega^{4}+2 u^{2} \omega^{2}\right) \\
& +\frac{\lambda_{2}}{4} v^{4}+\frac{\lambda_{3}}{4}\left(u^{2} v^{2}+v^{2} \omega^{2}\right) .
\end{aligned}
$$

We see that $V_{0}(u, \omega, v)$ has a quartic form like in the SM, but it depends on three variables, $u, \omega$ and $v$; it also has mixings between these variables. However, we can transform $u$ into $\omega$ by $t_{\theta}$ as defined in Eq. (17). We note that if the Universe's energies allow for the existence of the gauge symmetry $S U(3)_{L} \otimes U(1)_{X}$ and the SSB sequence in the E331 model, the VEVs $u, \omega$ and $v$ must satisfy the constraint (4). This leads to $t_{\theta} \ll 1$, and we can neglect the contribution of $u$. On the other hand, by developing the Higgs potential (1), we obtain two minimum equations which permit us to transform the mixing between $\omega$ and $v$,

$\mu_{1}^{2}+\lambda_{1}\left(u^{2}+\omega^{2}\right)+\lambda_{3} \frac{v^{2}}{2}=0$,

$\mu_{2}^{2}+\lambda_{2} v^{2}+\lambda_{3} \frac{\left(u^{2}+\omega^{2}\right)}{2}=0$.

From Eq. (29) we obtain

$\lambda_{3}\left(u^{2}+\omega^{2}\right) v^{2}=-2\left(\mu_{2}^{2} v^{2}+\lambda_{2} v^{4}\right)$,

and

$u^{2} \omega^{2}=\frac{\mu_{2}^{2}-\frac{2 \lambda_{2}}{\lambda_{3}} \mu_{1}^{2}}{\frac{2 \lambda_{1} \lambda_{2}}{\lambda_{3}}-\frac{\lambda_{3}}{2}} u^{2}-u^{4}$.
Substituting Eqs. (30) and (31) into Eq. (28) yields

$$
\begin{aligned}
& V_{0}(u, \omega, v)=\frac{\mu_{1}^{2}}{2} \omega^{2}+\frac{\lambda_{1}}{4} \omega^{4} \\
& +\left[\frac{\mu_{1}^{2}}{2}+2 \frac{\mu_{2}^{2}-\frac{2 \lambda_{2}}{\lambda_{3}} \mu_{1}^{2}}{\frac{2 \lambda_{1} \lambda_{2}}{\lambda_{3}}-\frac{\lambda_{3}}{2}}\right] u^{2}-\frac{\lambda_{1}}{4} u^{4}-\frac{\lambda_{2} v^{4}}{4} .
\end{aligned}
$$

Neglecting $u$ and from those relations, we can write $V_{0}$ in Eq. (32) as a sum of two parts corresponding to two stages of SSB:

$V_{0}(\omega, v)=V_{0}(\omega)+V_{0}(v)$

where $V_{0}(\omega)=\frac{\mu_{1}^{2}}{2} \omega^{2}+\frac{\lambda_{1}}{4} \omega^{4}$ and $V_{0}(v)=-\frac{\lambda_{2} v^{4}}{4}$ are in the quartic form. In addition, we have alternative ways to arrive Eq. (33) which has other forms but $V_{0}(\omega)$ and $V_{0}(v)$ are still in the quartic form.

In order to derive the effective potential, we start from the full Higgs Lagrangian:

$\mathcal{L}=\mathcal{L}_{\text {mass }}^{G B}+V(\chi, \phi)$,

where $\mathcal{L}_{\text {mass }}^{G B}$ and $V(\chi, \phi)$ are respectively given by Eq. (9) and Eq. (1).

In order to see the effective potential of this model can be split into two separated parts, we analyze the processes which generate the masses for all particles. The first, we want to mention the masses of the gauge fields which come from Eq. (9), we can rewrite this equation as form:

$\mathcal{L}_{\text {mass }}^{G B}=\left(\mathcal{D}_{\mu}\langle\chi\rangle\right)^{\dagger}\left(\mathcal{D}^{\mu}\langle\chi\rangle\right)+\left(\mathcal{D}_{\mu}\langle\phi\rangle\right)^{\dagger}\left(\mathcal{D}^{\mu}\langle\phi\rangle\right)=A+B$,

where

$A \equiv\left(\mathcal{D}_{\mu}\langle\chi\rangle\right)^{\dagger}\left(\mathcal{D}^{\mu}\langle\chi\rangle\right)$,
$B \equiv\left(\mathcal{D}_{\mu}\langle\phi\rangle\right)^{\dagger}\left(\mathcal{D}_{\mu}\langle\phi\rangle\right)$.

Note that the gauge fields $\left(W_{i}^{\mu}, B^{\mu}\right)$ inside the covariant derivatives of the $A$ and $B$ are the same. So after diagonalizing, the gauge fields in the $A$ and $B$ are the same, and we obtain gauge bosons: $\gamma, Z_{1}, Z_{2}, X_{0}, W^{ \pm}, Y^{ \pm}$.

Neglecting $u$ and from the term $A$, one obtains the mass components of the physical gauge bosons only depend on $\omega$ and $u$ (for details see, the second paper in [38-44]):

$$
\begin{aligned}
& M_{\text {bosons }}^{A}=m_{W^{ \pm}}^{2}(\omega, u) W_{\mu}^{+} W^{-\mu}+m_{Y^{ \pm}}^{2}(\omega, u) Y_{\mu}^{+} Y^{-\mu} \\
& \quad+m_{X^{0}}^{2}(\omega, u) X_{\mu}^{0} Z^{0 \mu} \\
& \quad+m_{Z^{1}}^{2}(\omega, u) Z_{\mu}^{1} Z^{1 \mu}+m_{Z^{2}}^{2}(\omega, u) Z_{\mu}^{2} Z^{2 \mu} .
\end{aligned}
$$


From the term $B$, one obtains the mass components of the physical gauge bosons only depend on $v$ :

$$
\begin{aligned}
& M_{\text {bosons }}^{B}=m_{W^{ \pm}}^{2}(v) W_{\mu}^{+} W^{-\mu}+m_{Y^{ \pm}}^{2}(v) Y_{\mu}^{+} Y^{-\mu} \\
& \quad+m_{X^{0}}^{2}(v) X_{\mu}^{0} Z^{0 \mu}+m_{Z^{1}}^{2}(v) Z_{\mu}^{1} Z^{1 \mu} \\
& \quad+m_{Z^{2}}^{2}(v) Z_{\mu}^{2} Z^{2 \mu} .
\end{aligned}
$$

Looking carefully at Eq. (14) and (19), this is easily checked. So we obtain $m_{W^{ \pm}}^{2}(\omega, u)=m_{X^{0}}^{2}(v)=0$, $m_{Z_{1}}^{2}(\omega, u)=m_{Z_{2}}^{2}(v) \approx 0$.

Through this analysis we see that when we combine A with $\mathrm{B}$ to make the diagonalization of the matrix in the above section, this leads to a mixing between the gauge bosons in the $A$ and $B$. However, we note that although the fields of the $A$ and $B$ are the same, the fields in the $A$ or Eq. (36) only go with $\omega$ and $u$, the fields in the $B$ or Eq. (37) only go with $v$. Because the $A$ and $B$ are not mix together. Therefore we found that the physical gauge bosons are like "break", this problem is due to the initial assumptions of the model about the covariant derivative.

The second, we want to mention the masses of the Higgs fields. The Higgs potential, Eq. (1), has two last components, $\lambda_{3}\left(\chi^{\dagger} \chi\right)\left(\phi^{\dagger} \phi\right)$ and $\lambda_{4}\left(\chi^{\dagger} \phi\right)\left(\phi^{\dagger} \chi\right)$, so we have a mixing among VEVs. In the calculation in the above section, we perform an approximation in Eqs. (6)-(8). These approximations did lose the mixing between VEVs, so they did lose $\lambda_{3}\left(\chi^{\dagger} \chi\right)\left(\phi^{\dagger} \phi\right)$ and $\lambda_{4}\left(\chi^{\dagger} \phi\right)\left(\phi^{\dagger} \chi\right)$ or these two last components are absorbed into the other components of the Higgs potential.

Therefore the masses of the gauge bosons and the Higgses presented in Table 1, from which we can split the boson masses into two parts for two SSB stages:

$m_{\text {boson }}^{2}(\omega, v)=m_{\text {boson }}^{2}(\omega)+m_{\text {boson }}^{2}(v)$.

However, note that the Higgs fields are like the gauge bosons. A field function multiplies by the square of the mass that contains a VEV, so that the field only effects on the $\mathrm{VEV}$, but it does not effect on all of VEVs.

The last, expanding the Higgs fields $\chi$ and $\phi$ around their VEVs which are $u, \omega$ and $v$, we obtain

$$
\begin{aligned}
\mathcal{L}= & \frac{1}{2} \partial^{\mu} \omega \partial_{\mu} \omega+\frac{1}{2} \partial^{\mu} v \partial_{\mu} v+V_{0}(\omega, v)+M_{\text {bosons }}^{A}+M_{\text {bosons }}^{B} \\
& +\sum m_{\text {exotic quarks }}(\omega) Q \bar{Q}+m_{\text {top quark }}(v) t \bar{t}
\end{aligned}
$$

In the E331 model, we have two massive bosons like the SM bosons $Z_{1}$ and $W^{ \pm}$, two new heavy neutral bosons $X_{0}$ and $Z_{2}$, the singly charged gauge bosons $Y^{ \pm}$, one singly charged Higgs $H_{2}^{ \pm}$, one heavy neutral Higgs $H_{1}^{0}$ and one SM-like Higgs $H^{0}$. We must consider contributions from all fermions and bosons. But for fermions, we retain only the top and exotic quarks because their contributions dominate over those from the other fermions [2]. Therefore, from the Lagrangain (39) we obtain two motion equations according to $\omega$ and $v$,

$$
\begin{gathered}
\partial^{\mu} \omega \partial_{\mu} \omega+\frac{\partial V_{0}(\omega)}{\partial \omega}+\sum \frac{\partial m_{\text {bosons }}^{2}(\omega)}{\partial \omega} W^{\mu} W_{\mu} \\
+\sum \frac{\partial m_{\text {exotic quarks }}(\omega)}{\partial \omega} Q \bar{Q}=0, \\
\partial^{\mu} v \partial_{\mu} v+\frac{\partial V_{0}(v)}{\partial v}+\sum \frac{\partial m_{\text {bosons }}^{2}(v)}{\partial v} W^{\mu} W_{\mu} \\
+\frac{\partial m_{\text {top quark }}(v)}{\partial v} t \bar{t}=0,
\end{gathered}
$$

where $W$ runs over all gauge fields and Higgs bosons. From Eq. (42), averaging over space we obtain

$$
\begin{gathered}
\partial^{\mu} \omega \partial_{\mu} \omega+\frac{\partial V_{0}(\omega)}{\partial \omega}+\sum \frac{\partial m_{\text {bosons }}^{2}(\omega)}{\partial \omega}\left\langle W^{\mu} W_{\mu}\right\rangle \\
+\sum \frac{\partial m_{\text {exotic quarks }}(\omega)}{\partial \omega}\langle Q \bar{Q}\rangle=0, \\
\partial^{\mu} v \partial_{\mu} v+\frac{\partial V_{0}(v)}{\partial v}+\sum \frac{\partial m_{\text {bosons }}^{2}(v)}{\partial v}\left\langle W^{\mu} W_{\mu}\right\rangle \\
+\frac{\partial m_{\text {top quark }}(v)}{\partial v}\langle t \bar{t}\rangle=0 .
\end{gathered}
$$

Note that $\left\langle W^{\mu} W_{\mu}\right\rangle$ in Eq. (42) only effect on $\omega$, so it only depends on $m_{\text {bosons }}(\omega)$. Similarly, $\left\langle W^{\mu} W_{\mu}\right\rangle$ in Eq. (43) only effect on $v$, so it only depends on $m_{\text {bosons }}(v)$.

Using Bose-Einstein and Fermi-Dirac distributions respectively for bosons and fermions to average over space, we obtain the one-loop effective potential $V_{\text {eff }}(\omega)$ for the electroweak phase transition $S U(3)-S U(2)$ at high temperatures:

$$
\begin{aligned}
& V_{\mathrm{eff}}(\omega)=V_{0}(\omega) \\
& +\frac{1}{64 \pi^{2}}\left[6 m_{Y}^{4}(\omega) \ln \frac{m_{Y}^{2}(\omega)}{Q^{\prime 2}}+6 m_{X}^{4}(\omega) \ln \frac{m_{X}^{2}(\omega)}{Q^{\prime 2}}\right. \\
& +3 m_{Z_{2}}^{4}(\omega) \ln \frac{m_{Z_{2}}^{2}(\omega)}{Q^{\prime 2}}+m_{H_{1}^{0}}^{4}(\omega) \ln \frac{m_{H_{1}^{0}}^{2}(\omega)}{Q^{\prime 2}} \\
& \left.+2 m_{H_{2}^{+}}^{4}(\omega) \ln \frac{m_{H_{2}^{+}}^{2}(\omega)}{Q^{\prime 2}}-36 m_{Q}^{4}(\omega) \ln \frac{m_{Q}^{2}(\omega)}{Q^{\prime 2}}\right] \\
& +\frac{T^{4}}{4 \pi^{2}}\left[6 F_{-}\left(\frac{m_{Y}(\omega)}{T}\right)+6 F_{-}\left(\frac{m_{X}(\omega)}{T}\right)\right. \\
& +3 F_{-}\left(\frac{m_{Z_{2}}(\omega)}{T}\right)+F_{-}\left(\frac{m_{H_{1}^{0}}(\omega)}{T}\right) \\
& \left.+2 F_{-}\left(\frac{m_{H_{2}^{+}}(\omega)}{T}\right)+36 F_{+}\left(\frac{m_{Q}(\omega)}{T}\right)\right]
\end{aligned}
$$


Table 1 Mass formulations of bosons in the E331 model

\begin{tabular}{|c|c|c|c|c|}
\hline Bosons & $m^{2}(\omega, v)$ & $m^{2}(\omega)$ & $m^{2}(v)$ & $m^{2}\left(v_{0}=246 \mathrm{GeV}\right)$ \\
\hline$m_{W^{ \pm}}^{2}$ & $\frac{g^{2}}{4} v^{2}$ & 0 & $\frac{g^{2}}{4} v^{2}$ & $80.39^{2}(\mathrm{GeV})^{2}$ \\
\hline$m_{Y^{ \pm}}^{2}$ & $\frac{g^{2}}{4}\left(\omega^{2}+v^{2}\right)$ & $\frac{g^{2}}{4} \omega^{2}$ & $\frac{g^{2}}{4} v^{2}$ & $80.39^{2}(\mathrm{GeV})^{2}$ \\
\hline$m_{X^{0}}^{2}$ & $\frac{g^{2}}{4} \omega^{2}$ & $\frac{g^{2}}{4} \omega^{2}$ & 0 & 0 \\
\hline$m_{Z_{1}}^{2} \sim m_{Z}^{2}$ & $\frac{g^{2}}{4 c_{W}^{2}} v^{2}$ & 0 & $\frac{g^{2}}{4 c_{W}^{2}} v^{2}$ & $91.68^{2}(\mathrm{GeV})^{2}$ \\
\hline$m_{Z_{2}}^{2} \sim m_{Z^{\prime}}^{2}$ & $\frac{g^{2} c_{W}^{2}}{3-4 s_{W}^{2}} \omega^{2}$ & $\frac{g^{2} c_{W}^{2}}{3-4 s_{W}^{2}} \omega^{2}$ & 0 & 0 \\
\hline$m_{H^{0}}^{2}$ & $\left(2 \lambda_{2}-\frac{\lambda_{3}^{2}}{2 \lambda_{1}}\right) v^{2}$ & 0 & $\left(2 \lambda_{2}-\frac{\lambda_{3}^{2}}{2 \lambda_{1}}\right) v^{2}$ & $125^{2}(\mathrm{GeV})^{2}$ \\
\hline$m_{H_{1}^{0}}^{2}$ & $2 \lambda_{1} \omega^{2}+\frac{\lambda_{3}^{2}}{2 \lambda_{1}} v^{2}$ & $2 \lambda_{1} \omega^{2}$ & $\frac{\lambda_{3}^{2}}{2 \lambda_{1}} v^{2}$ & $\frac{\lambda_{3}^{2}}{2 \lambda_{1}} v_{0}^{2}$ \\
\hline$m_{H_{2}^{ \pm}}^{2}$ & $\frac{\lambda_{4}}{2}\left(\omega^{2}+v^{2}\right)$ & $\frac{\lambda_{4}}{2} \omega^{2}$ & $\frac{\lambda_{4}}{2} v^{2}$ & $\frac{\lambda_{4}}{2} v_{0}^{2}$ \\
\hline
\end{tabular}

in which $m_{Q}$ indicates the masses of three exotic quarks. Similarly, from Eq. (43), we obtain the high-temperature effective potential $V_{\text {eff }}(v)$ for the electroweak phase transition $S U(2)-$ $U(1)$ :

$$
\begin{aligned}
& V_{\text {eff }}(v)=V_{0}(v) \\
& +\frac{1}{64 \pi^{2}}\left[6 m_{W}^{4}(v) \ln \frac{m_{W}^{2}(v)}{Q^{2}}+6 m_{Y}^{4}(v) \ln \frac{m_{Y}^{2}(v)}{Q^{2}}\right. \\
& +3 m_{Z_{1}}^{4}(v) \ln \frac{m_{Z_{1}}^{2}(v)}{Q^{2}}+m_{H^{0}}^{4}(v) \ln \frac{m_{H^{0}}^{2}(v)}{Q^{2}} \\
& +m_{H_{1}^{4}}^{4}(v) \ln \frac{m_{H_{1}^{0}}^{2}(v)}{Q^{2}} \\
& \left.+2 m_{H_{2}^{+}}^{4}(v) \ln \frac{m_{H_{2}^{+}}^{2}(v)}{Q^{2}}-12 m_{t}^{4}(v) \ln \frac{m_{t}^{2}(v)}{Q^{2}}\right] \\
& +\frac{T^{4}}{4 \pi^{2}}\left[6 F_{-}\left(\frac{m_{W}(v)}{T}\right)+6 F_{-}\left(\frac{m_{Y}(v)}{T}\right)\right. \\
& +3 F_{-}\left(\frac{m_{Z_{1}}(v)}{T}\right)+F_{-}\left(\frac{m_{H^{0}}(v)}{T}\right)+F_{-}\left(\frac{m_{H_{1}^{0}}(v)}{T}\right) \\
& \left.+2 F_{-}\left(\frac{m_{H_{2}^{+}}(v)}{T}\right)+12 F_{+}\left(\frac{m_{t}(v)}{T}\right)\right]
\end{aligned}
$$

in which $m_{t}$ indicates the mass of the top quark. $F_{\mp}\left(\frac{m}{T}\right)$ come from $\left\langle W^{\mu} W_{\mu}\right\rangle$ and describe the thermal contributions of particles with masses $m$. These terms are given by

$F_{\mp}\left(\frac{m}{T}\right)=\int_{0}^{\frac{m}{T}} \alpha J_{\mp}^{(1)}(\alpha, 0) \mathrm{d} \alpha$,

where

$J_{\mp}^{(1)}(\alpha, 0)=2 \int_{\alpha}^{\infty} \frac{\left(x^{2}-\alpha^{2}\right)^{1 / 2}}{e^{x} \mp 1} \mathrm{~d} x$.
As the above analyze, $F_{\mp}\left(\frac{m}{T}\right)$ in Eq. (44) only depend on $\omega$ and $F_{\mp}\left(\frac{m}{T}\right)$ in Eq. (45) only depend on $v$.

Equations (39)-(44) and (45) do not consist of any mixing between $\omega$ and $v$. Therefore, we can write the total effective potential in the E331 model as

$V_{\mathrm{eff}}^{\mathrm{E} 331}=V_{\mathrm{eff}}(\omega)+V_{\mathrm{eff}}(v)$

The effective potentials $V_{\text {eff }}(\omega)$ and $V_{\text {eff }}(v)$ seem to depend on the arbitrary scales $Q^{\prime}$ and $Q$ respectively. However, by the same reasoning as in [2], we can show that the structure of these potentials remain unchanged for the changes in scales. At zero temperature, all thermal contributions vanish, and due to the quartic form of $V_{0}(\omega)$ and $V_{0}(v)$, we can rewrite Eqs. (44) and (45) as

$$
\begin{aligned}
& V_{\mathrm{eff}}^{0^{o} K}(\omega)=\lambda_{R}^{\prime} \omega^{4}+M_{R}^{\prime 2} \omega^{2}+\Lambda_{R}^{\prime}+\frac{1}{64 \pi^{2}} \\
& \times\left[6 m_{Y}^{4}(\omega) \ln \frac{m_{Y}^{2}(\omega)}{Q^{\prime 2}}+6 m_{X}^{4}(\omega) \ln \frac{m_{X}^{2}(\omega)}{Q^{\prime 2}}\right. \\
& +3 m_{Z_{2}}^{4}(\omega) \ln \frac{m_{Z_{2}}^{2}(\omega)}{Q^{\prime 2}}+m_{H_{1}^{0}}^{4}(\omega) \ln \frac{m_{H_{1}^{0}}^{2}(\omega)}{Q^{\prime 2}} \\
& \left.\quad+2 m_{H_{2}^{+}}^{4}(\omega) \ln \frac{m_{H_{2}^{+}}^{2}(\omega)}{Q^{\prime 2}}-36 m_{Q}^{4}(\omega) \ln \frac{m_{Q}^{2}(\omega)}{Q^{\prime 2}}\right]
\end{aligned}
$$

and

$$
\begin{aligned}
& V_{\mathrm{eff}}^{0^{o} K}(v)=\lambda_{R} v^{4}+M_{R}^{2} v^{2}+\Lambda_{R}+\frac{1}{64 \pi^{2}} \\
& \times\left[6 m_{W}^{4}(v) \ln \frac{m_{W}^{2}(v)}{Q^{2}}+6 m_{Y}^{4}(v) \ln \frac{m_{Y}^{2}(v)}{Q^{2}}\right. \\
& +3 m_{Z_{1}}^{4}(v) \ln \frac{m_{Z_{1}}^{2}(v)}{Q^{2}}+m_{H^{0}}^{4}(v) \ln \frac{m_{H^{0}}^{2}(v)}{Q^{2}}
\end{aligned}
$$




$$
\begin{aligned}
& +m_{H_{1}^{0}}^{4}(v) \ln \frac{m_{H_{1}^{0}}^{2}(v)}{Q^{2}} \\
& \left.+2 m_{H_{2}^{+}}^{4}(v) \ln \frac{m_{H_{2}^{+}}^{2}(v)}{Q^{2}}-12 m_{t}^{4}(v) \ln \frac{m_{t}^{2}(v)}{Q^{2}}\right],
\end{aligned}
$$

where $\lambda_{R}^{\prime}, M_{R}^{\prime}, \Lambda_{R}^{\prime}, \lambda_{R}, M_{R}$, and $\Lambda_{R}$ are the renormalized constants. The changes such as $Q^{\prime} \rightarrow \kappa^{\prime} Q^{\prime}$ (or $Q \rightarrow \kappa Q$ ) induce the terms which contain $\kappa^{\prime}$ (or $\kappa$ ) and are proportional to $m_{\text {boson }}^{4}(\omega) \sim \omega^{4}$ (or $m_{\text {boson }}^{4}(v) \sim v^{4}$ ). Those terms can be absorbed by $\lambda_{R}^{\prime}$ (or $\lambda_{R}$ ). This makes the physics remain the same.

For this reason, we can put $Q^{\prime}=\epsilon^{\prime} \omega_{0}$ and $Q=\epsilon v_{0}$ into Eqs. (49) and (50), respectively. Combining the terms which contain $\epsilon^{\prime}$ and $\epsilon$ with the renormalized constants, we have

$$
\begin{gathered}
V_{\mathrm{eff}}^{0^{o} K}(\omega)=\frac{\lambda_{0}^{\prime}}{4} \omega^{4}+M_{0}^{\prime 2} \omega^{2}+\Lambda_{0}^{\prime}+\frac{1}{64 \pi^{2}} \\
\times\left[6 m_{Y}^{4}(\omega) \ln \frac{\omega^{2}}{\omega_{0}^{2}}+6 m_{X}^{4}(\omega) \ln \frac{\omega^{2}}{\omega_{0}^{2}}\right. \\
+3 m_{Z_{2}}^{4}(\omega) \ln \frac{\omega^{2}}{\omega_{0}^{2}}+m_{H_{1}^{0}}^{4}(\omega) \ln \frac{\omega^{2}}{\omega_{0}^{2}} \\
\left.+2 m_{H_{2}^{+}}^{4}(\omega) \ln \frac{\omega^{2}}{\omega_{0}^{2}}-36 m_{Q}^{4}(\omega) \ln \frac{\omega^{2}}{\omega_{0}^{2}}\right]
\end{gathered}
$$

and

$$
\begin{gathered}
V_{\mathrm{eff}}^{0^{o} K}(v)=\frac{\lambda_{0}}{4} v^{4}+M_{0}^{2} v^{2}+\Lambda_{0}+\frac{1}{64 \pi^{2}} \\
\times\left[6 m_{W}^{4}(v) \ln \frac{v^{2}}{v_{0}^{2}}+6 m_{Y}^{4}(v) \ln \frac{v^{2}}{v_{0}^{2}}\right. \\
+3 m_{Z_{1}}^{4}(v) \ln \frac{v^{2}}{v_{0}^{2}}+m_{H^{0}}^{4}(v) \ln \frac{v^{2}}{\left(v_{0}\right)^{2}} \\
+m_{H_{1}^{0}}^{4}(v) \ln \frac{v^{2}}{v_{0}^{2}} \\
\left.\quad+2 m_{H_{2}^{+}}^{4}(v) \ln \frac{v^{2}}{v_{0}^{2}}-12 m_{t}^{4}(v) \ln \frac{v^{2}}{v_{0}^{2}}\right],
\end{gathered}
$$

where $\lambda_{0}^{\prime}, M_{0}^{\prime 2}, \Lambda_{0}^{\prime}, \lambda_{0}, M_{0}^{2}, \Lambda_{0}$ are the parameters those can be specified from the conditions (61) and (67). We obtain

$$
\begin{aligned}
\lambda_{0}^{\prime}= & \left\{\frac{m_{H_{1}^{0}}^{2}\left(\omega_{0}\right)}{2 \omega_{0}^{2}}-\frac{3}{32 \pi^{2}}\left(6 m_{Y}^{4}\left(\omega_{0}\right)+6 m_{X}^{4}\left(\omega_{0}\right)\right.\right. \\
& \left.\left.+3 m_{Z_{2}}^{4}\left(\omega_{0}\right)+m_{H_{1}^{0}}^{4}\left(\omega_{0}\right)+2 m_{H_{2}^{+}}^{4}\left(\omega_{0}\right)-36 m_{Q}^{4}\left(\omega_{0}\right)\right)\right\},
\end{aligned}
$$

$$
\begin{aligned}
M_{0}^{\prime 2}= & \left\{-\frac{1}{4} m_{H_{1}^{0}}^{2}\left(\omega_{0}\right)+\frac{1}{32 \pi^{2} \omega_{0}^{2}}\left(6 m_{Y}^{4}\left(\omega_{0}\right)+6 m_{X}^{4}\left(\omega_{0}\right)\right.\right. \\
& \left.\left.+3 m_{Z_{2}}^{4}\left(\omega_{0}\right)+m_{H_{1}^{0}}^{4}\left(\omega_{0}\right)+2 m_{H_{2}^{+}}^{4}\left(\omega_{0}\right)-36 m_{Q}^{4}\left(\omega_{0}\right)\right)\right\}, \\
\Lambda_{0}^{\prime} & =\frac{\omega_{0}^{2}}{4}\left\{\frac{m_{H_{1}^{0}}^{2}\left(\omega_{0}\right)}{2}-\frac{1}{32 \pi^{2} \omega_{0}^{2}}\left(6 m_{Y}^{4}\left(\omega_{0}\right)+6 m_{X}^{4}\left(\omega_{0}\right)\right.\right. \\
& \left.\left.+3 m_{Z_{2}}^{4}\left(\omega_{0}\right)+m_{H_{1}^{0}}^{4}\left(\omega_{0}\right)+2 m_{H_{2}^{+}}^{4}\left(\omega_{0}\right)-36 m_{Q^{4}}^{4}\left(\omega_{0}\right)\right)\right\} ;
\end{aligned}
$$

$$
\begin{aligned}
\lambda_{0} & =\left\{\frac{m_{H^{0}}^{2}\left(v_{0}\right)+m_{H_{1}^{0}}^{2}\left(v_{0}\right)}{2 v_{0}^{2}}-\frac{3}{32 \pi^{2}}\right. \\
& \times\left(6 m_{W}^{4}\left(v_{0}\right)+6 m_{Y}^{4}\left(v_{0}\right)+3 m_{Z_{1}}^{4}\left(v_{0}\right)\right. \\
& \left.\left.+m_{H^{0}}^{4}\left(v_{0}\right)+m_{H_{1}^{0}}^{4}\left(v_{0}\right)+2 m_{H_{2}^{+}}^{4}\left(v_{0}\right)-12 m_{t}^{4}\left(v_{0}\right)\right)\right\}, \\
M_{0}^{2} & =\left\{-\frac{m_{H}^{2}\left(v_{0}\right)+m_{H_{1}^{0}}\left(v_{0}\right)}{4}+\frac{1}{32 \pi^{2} v_{0}^{2}}\right. \\
& \times\left(6 m_{W}^{4}\left(v_{0}\right)+6 m_{Y}^{4}\left(v_{0}\right)+3 m_{Z_{1}}^{4}\left(v_{0}\right)\right. \\
& \left.\left.+m_{H^{0}}^{4}\left(v_{0}\right)+m_{H_{1}^{0}}^{4}\left(v_{0}\right)+2 m_{H_{2}^{+}}^{4}\left(v_{0}\right)-12 m_{t}^{4}\left(v_{0}\right)\right)\right\},
\end{aligned}
$$$$
\Lambda_{0}=\frac{v_{0}^{2}}{4}\left\{\frac{m_{H^{0}}^{2}\left(v_{0}\right)+m_{H_{1}^{0}}^{2}\left(v_{0}\right)}{2}-\frac{1}{32 \pi^{2} v_{0}^{2}}\right.
$$$$
\times\left(6 m_{W}^{4}\left(v_{0}\right)+6 m_{Y}^{4}\left(v_{0}\right)+3 m_{Z_{1}}^{4}\left(v_{0}\right)\right.
$$$$
\left.\left.+m_{H^{0}}^{4}\left(v_{0}\right)+m_{H_{1}^{0}}^{4}\left(v_{0}\right)+2 m_{H_{2}^{+}}^{4}\left(v_{0}\right)-12 m_{t}^{4}\left(v_{0}\right)\right)\right\} \text {. }
$$

In the special case, when $M_{0}^{2}=0$, the potential (52) reduces to the Coleman-Weinberg potential.

\section{Electroweak phase transition}

In sequence of SSB of the E331 model, the SSB which breaks the gauge symmetry $S U(3)_{L} \otimes U(1)_{X}$ down to the $S U(2)_{L} \otimes$ $U(1)_{Y}$ through $\chi_{3}^{0}$ generates the masses for the exotic quarks, the heavy gauge bosons $X^{0}$ and $Z_{2}$, and gives the first part of mass for $Y^{ \pm}$. The SSB which breaks the symmetry $S U(2)_{L} \otimes$ $U(1)_{Y}$ down to the $U(1)_{Q}$ through $\chi_{1}^{0}$ and $\phi_{2}^{0}$ generates the 
masses for the SM particles and gives the last part of mass for $Y^{ \pm}$. Because $\omega_{0} \sim \mathcal{O}(1) \mathrm{TeV}, u_{0} \sim \mathcal{O}(1) \mathrm{GeV}$, and $v_{0}=246 \mathrm{GeV}[38-44,46]$, the breaking $S U(3) \rightarrow S U(2)$ occurs before the breaking $S U(2) \rightarrow U(1)$.

Associated with this sequence of SSB, a sequence of EWPT takes place with the transition $S U(3) \rightarrow S U(2)$ at the scale of $\omega_{0}$ and the transition $S U(2) \rightarrow U(1)$ at the scale of $v_{0}$ as the Universe cools down from the hot big bang. Our analysis so far shows that the former is the first transition which depends only on $\omega$, while the latter is the second transition which depends only on $v$.

From Table 1, the gauge bosons $X^{0}$ and $Z_{2}$ are only involved in the first transition, the gauge bosons $W^{ \pm}, Z_{1}$ and $H^{0}$ are only involved in the second transition, but the bosons $Y^{ \pm}, H_{1}^{0}$, and $H_{2}^{+}$are involved in both transitions. The total mass of $Y^{ \pm}$-i.e. $m_{Y^{ \pm}}(\omega, v)$, whose formula is given by (38) - is generated as follows. As the Universe is at the $\omega_{0}$ scale and the EWPT $S U(3) \rightarrow S U(2)$ happens, $Y^{ \pm}$eats the Goldstone boson $\chi_{2}^{ \pm}$of the triplet $\chi$ to obtain the first part of mass, $m_{Y^{ \pm}}(\omega)$. When the Universe cools to the $v_{0}$ scale and the EWPT $S U(2) \rightarrow U(1)$ is turned on, $Y^{ \pm}$ eats the Goldstone boson $\rho_{1}^{ \pm}$of triplet $\phi$ and get the last part of mass, $m_{Y^{ \pm}}(v)$.

\subsection{Phase transition $S U(3) \rightarrow S U(2)$}

Taking place at the scale of $\omega_{0}$, which is chosen to be in the range $1-5 \mathrm{TeV}$, the EWPT $S U(3) \rightarrow S U(2)$ involves exotic quarks and heavy bosons, without the involvement of the SM particles. From Eq. (44), the high-temperature effective potential of the EWPT can be rewritten as

$V_{\mathrm{eff}}(\omega)=D^{\prime}\left(T^{2}-T_{0}^{\prime 2}\right) \omega^{2}-E^{\prime} T \omega^{3}+\frac{\lambda_{T}^{\prime}}{4} \omega^{4}$,

in which

$$
\begin{aligned}
D^{\prime}= & \frac{1}{24 \omega_{0}^{2}}\left\{6 m_{Y}^{2}\left(\omega_{0}\right)+6 m_{X}^{2}\left(\omega_{0}\right)+3 m_{Z_{2}}^{2}\left(\omega_{0}\right)\right. \\
& \left.+m_{H_{1}^{0}}^{2}\left(\omega_{0}\right)+2 m_{H_{2}^{+}}^{2}\left(\omega_{0}\right)+18 m_{Q}^{2}\left(\omega_{0}\right)\right\}, \\
T_{0}^{\prime 2}= & \frac{1}{D^{\prime}}\left\{\frac{1}{4} m_{H_{1}^{0}}^{2}\left(\omega_{0}\right)-\frac{1}{32 \pi^{2} \omega_{0}^{2}}\left(6 m_{Y}^{4}\left(\omega_{0}\right)+6 m_{X}^{4}\left(\omega_{0}\right)\right.\right. \\
& \left.\left.+3 m_{Z_{2}}^{4}\left(\omega_{0}\right)+m_{H_{1}^{0}}^{4}\left(\omega_{0}\right)+2 m_{H_{2}^{+}}^{4}\left(\omega_{0}\right)-36 m_{Q^{4}}^{4}\left(\omega_{0}\right)\right)\right\}, \\
E^{\prime}= & \frac{1}{12 \pi \omega_{0}^{3}}\left(6 m_{Y}^{3}\left(\omega_{0}\right)+6 m_{X}^{3}\left(\omega_{0}\right)+3 m_{Z_{2}}^{3}\left(\omega_{0}\right)\right. \\
& \left.+m_{H_{1}^{0}}^{3}\left(\omega_{0}\right)+2 m_{H_{2}^{+}}^{3}\left(\omega_{0}\right)\right), \\
& m_{H_{1}^{0}}^{2}\left(\omega_{0}\right) \\
\lambda_{T}^{\prime}= & \frac{1}{2 \omega_{0}^{2}}\left\{1-\frac{m_{Y}^{2}\left(\omega_{0}\right)}{8 \pi^{2} \omega_{0}^{2} m_{H_{1}}^{2}\left(\omega_{0}\right)}\left[6 m_{Y}^{4}\left(\omega_{0}\right) \ln \frac{b T^{2}}{}\right.\right.
\end{aligned}
$$

$$
\begin{aligned}
& +6 m_{X}^{4}\left(\omega_{0}\right) \ln \frac{m_{X}^{2}\left(\omega_{0}\right)}{b T^{2}}+3 m_{Z_{2}}^{4}\left(\omega_{0}\right) \ln \frac{m_{Z_{2}}^{2}\left(\omega_{0}\right)}{b T^{2}} \\
& +m_{H_{1}^{0}}^{4}\left(\omega_{0}\right) \ln \frac{m_{H_{1}}^{2}\left(\omega_{0}\right)}{b T^{2}}+2 m_{H_{2}^{+}}^{4}\left(\omega_{0}\right) \ln \frac{m_{H_{2}^{+}}^{2}\left(\omega_{0}\right)}{b T^{2}} \\
& \left.\left.-36 m_{Q}^{4}\left(\omega_{0}\right) \ln \frac{m_{Q^{2}}^{2}\left(\omega_{0}\right)}{b_{F} T^{2}}\right]\right\}
\end{aligned}
$$

where $\omega_{0}$ is the value at which the zero-temperature effective potential $V_{\mathrm{eff}}^{0^{o} K}(\omega)$ gets the minimum. To obtain $V_{\mathrm{eff}}^{0^{o} K}(\omega)$, from $V_{\text {eff }}(\omega)$ in Eq. (44) we neglect all terms in the form $F_{\mp}\left(\frac{m}{T}\right)$. The minimum conditions for $V_{\mathrm{eff}}^{0^{o} K}(\omega)$ are:

$V_{\mathrm{eff}}^{0^{o} K}\left(\omega_{0}\right)=0 ;\left.\quad \frac{\partial V_{\mathrm{eff}}^{0^{o} K}(\omega)}{\partial \omega}\right|_{\omega=\omega_{0}}=0 ;$

$\left.\frac{\partial^{2} V_{\mathrm{eff}}^{0^{o} K}(\omega)}{\partial \omega^{2}}\right|_{\omega=\omega_{0}}=\left.m_{H_{1}^{0}}^{2}(\omega)\right|_{\omega=\omega_{0}}$.

From the conditions (61), we have the minima of the effective potential (59):

$\omega=0, \quad \omega \equiv \omega_{c}=\frac{2 E^{\prime} T_{c}^{\prime}}{\lambda_{T_{c}^{\prime}}^{\prime}}$,

where $\omega_{c}$ is a critical VEV of $\chi$ at the broken state, and $T_{c}^{\prime}$ is the critical temperature of the phase transition, which is given by

$T_{c}^{\prime}=\frac{T_{0}^{\prime}}{\sqrt{1-E^{\prime 2} / D^{\prime} \lambda_{T_{c}^{\prime}}^{\prime}}}$.

Now, we consider the phase-transition strength:

$S^{\prime}=\frac{\omega_{c}}{T_{c}^{\prime}}=\frac{2 E^{\prime}}{\lambda_{T_{c}^{\prime}}^{\prime}}$,

which is a function of three unknown masses, $m_{H_{1}^{0}}, m_{H_{2}^{ \pm}}$and $m_{Q}$. For simplicity, we follow the ansatz in [17] and assume $m_{H_{2}^{ \pm}}=m_{Q}$. Then we plot the transition strength $S^{\prime}$ as the function of $m_{H_{1}^{0}}\left(\omega_{c}\right)$ and $m_{H_{2}^{ \pm}}\left(\omega_{c}\right)$ with $\omega_{c}$ is in the range from 1 to $5 \mathrm{TeV}$. In Figs. 1, 2, 3, 4, and 5, we present the contours of $S^{\prime}$ in the $\left(m_{H_{2}^{ \pm}}, m_{H_{1}^{0}}\right)$-plane; each figure corresponds with a case of $\omega$. The smooth contours are the sets of the $\left(m_{H_{2}^{ \pm}}, m_{H_{1}^{0}}\right)$-pairs which make $S^{\prime}>1$ and then the EWPT $S U^{2}(3) \rightarrow S U(2)$ to be the first-order phase transition. The uneven contours are the sets of the $\left(m_{H_{2}^{ \pm}}, m_{H_{1}^{0}}\right)$ pairs which are unusable because they make $S^{\prime} \rightarrow \infty$. Our results show that the heavy particle masses must be in the range of a few $\mathrm{TeV}$, and the strength of the first-order phase transition $S U(3) \rightarrow S U(2)$ is in the range $1<S^{\prime}<13$.

According to Ref. [47], the accuracy of a high-temperature expansion for the effective potential such as that in Eq. (59) will be better than $5 \%$ if $\frac{m_{\text {boson }}}{T}<2.2$, where $m_{\text {boson }}$ is the relevant boson mass. This requirement sets the "upper bounds" of the mass ranges of $H_{1}^{0}(\omega)$ and $H_{2}^{ \pm}(\omega)$. From 


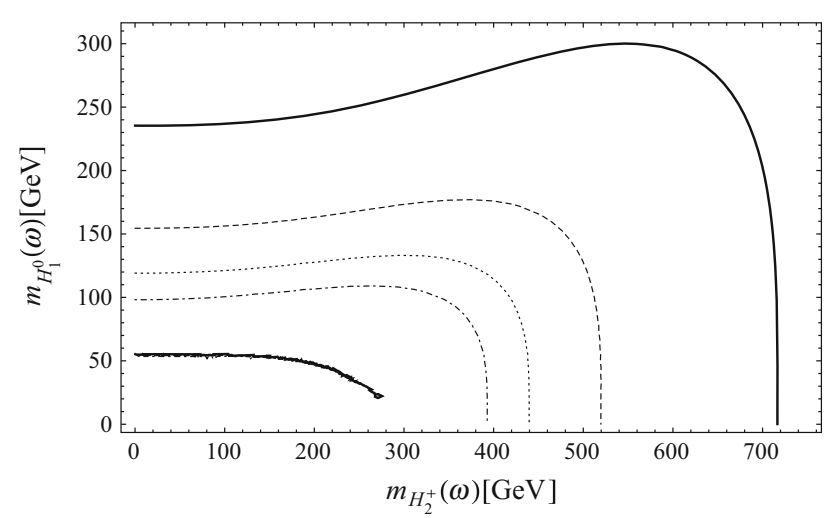

Fig. 1 The contours of $S^{\prime}=\frac{\omega_{c}}{T_{c}^{\prime}}$ in the case $\omega_{0}=1 \mathrm{TeV}$. Solid (and smooth) contour: $S^{\prime}=1$; dashed contour: $S^{\prime}=2$; dotted contour: $S^{\prime}=3$; dotted-dashed contour: $S^{\prime}=4$; uneven contour: $S^{\prime} \rightarrow \infty$. In this case, the mass ranges of $m_{H_{1}^{0}}$ and $m_{H^{ \pm}}$for the first-order phase transition are $0<m_{H_{1}^{0}}<300 \mathrm{GeV}$ and $0<m_{H_{2}^{ \pm}}<720 \mathrm{GeV}$, respectively

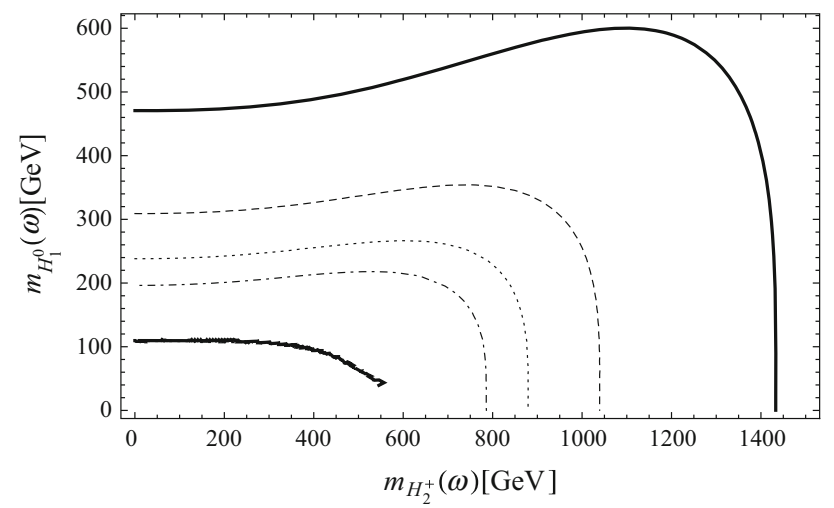

Fig. 2 The contours of $S^{\prime}=\frac{\omega_{c}}{T_{c}^{\prime}}$ in the case $\omega_{0}=2 \mathrm{TeV}$. Solid (and smooth) contour: $S^{\prime}=1$; dashed contour: $S^{\prime}=2$; dotted contour: $S^{\prime}=3$; dotted-dashed contour: $S^{\prime}=4$; uneven contour: $S^{\prime} \rightarrow \infty$. The mass ranges of $m_{H_{1}^{0}}$ and $m_{H_{2}^{ \pm}}$for the first-order phase transition are $0<m_{H_{1}^{0}}<600 \mathrm{GeV}$ and $0<m_{H_{2}^{ \pm}}<1440 \mathrm{GeV}$, respectively

Table 2, this requirement is satisfied by all mass ranges of $H_{1}^{0}$, while it narrows slightly most of the mass ranges of $H_{2}^{ \pm}$.

From Eq. (64), the phase-transition strength $S^{\prime}$ depends on the parameters $E^{\prime}$ and $\lambda_{T_{c}^{\prime}}^{\prime}$. From Eq. (60), $E^{\prime}$ expresses the contributions of the new bosons while $\lambda_{T_{c}^{\prime}}^{\prime}$ includes the contributions of the exotic quarks to the phase-transition strength. Therefore, the new bosons and exotic quarks can be triggers for the EWPT $S U(3) \rightarrow S U(2)$ to be the first-order.

\subsection{Phase transition $S U(2) \rightarrow U(1)$}

Occurring at the scale $v_{0}=246 \mathrm{GeV}$, the phase transition $S U(2) \rightarrow U(1)$ does not involve the exotic quarks or the boson $X^{0}$. In this stage, the contribution from $Y^{ \pm}$is equal to that from $W^{ \pm}$. The effective potential is given by Eq. (45). We write the high-temperature expansion of this potential as

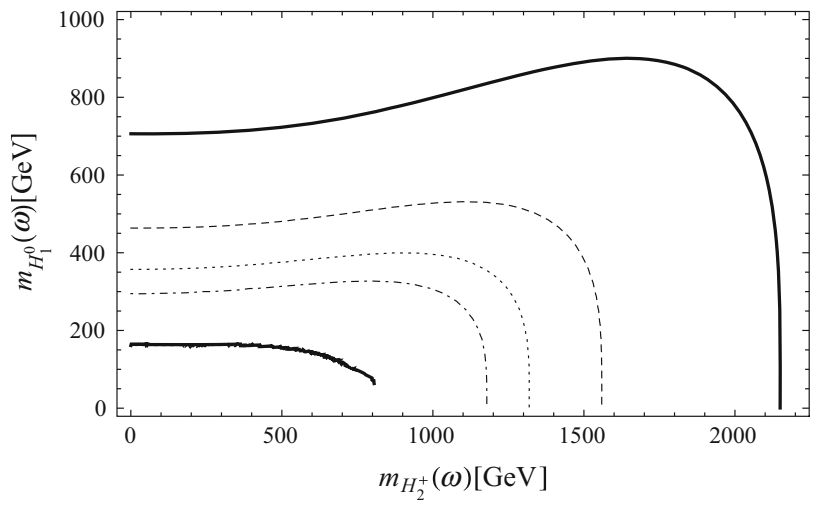

Fig. 3 The contours of $S^{\prime}=\frac{\omega_{c}}{T_{c}^{\prime}}$ in the case $\omega_{0}=3 \mathrm{TeV}$. Solid (and smooth) contour: $S^{\prime}=1$; dashed contour: $S^{\prime}=2$; dotted contour: $S^{\prime}=3$; dotted-dashed contour: $S^{\prime}=4$; uneven contour: $S^{\prime} \rightarrow \infty$. The mass ranges of $m_{H_{1}^{0}}$ and $m_{H_{2}^{ \pm}}$for the first-order phase transition are $0<m_{H_{1}^{0}}<900 \mathrm{GeV}$ and $0<m_{H_{2}^{ \pm}}<2150 \mathrm{GeV}$, respectively

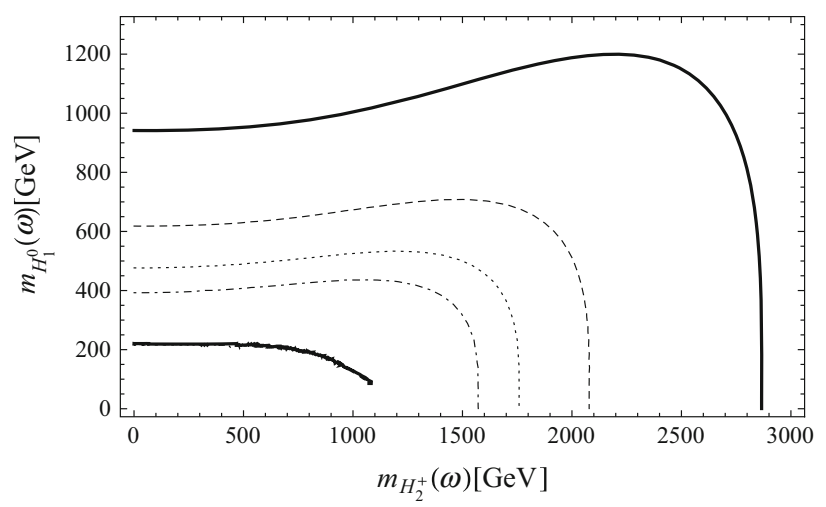

Fig. 4 The contours of $S^{\prime}=\frac{\omega_{c}}{T_{c}^{\prime}}$ in the case $\omega_{0}=4 \mathrm{TeV}$. Solid (and smooth) contour: $S^{\prime}=1$; dashed contour: $S^{\prime}=2$; dotted contour: $S^{\prime}=3$; dotted-dashed contour: $S^{\prime}=4$; uneven contour: $S^{\prime} \rightarrow \infty$. The mass ranges of $m_{H_{1}^{0}}$ and $m_{H_{2}^{ \pm}}$for the first-order phase transition are $0<m_{H_{1}^{0}}<1200 \mathrm{GeV}$ and $0<m_{H_{2}^{ \pm}}<2870 \mathrm{GeV}$, respectively

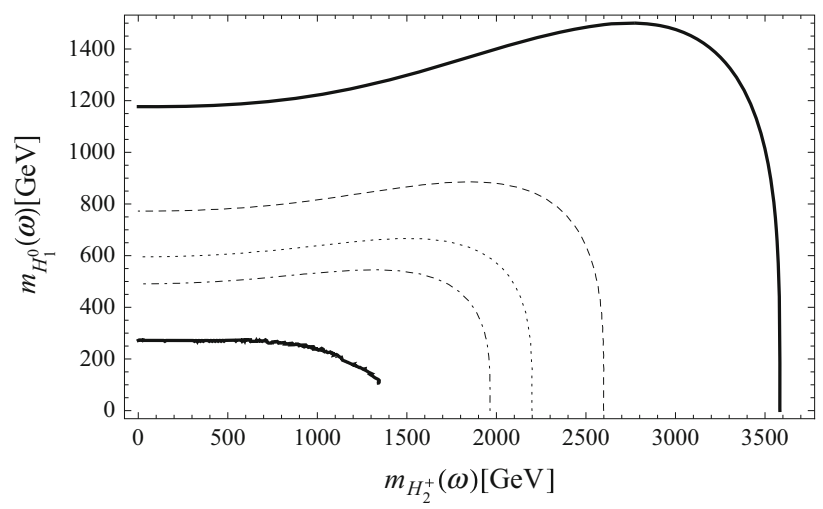

Fig. 5 The contours of $S^{\prime}=\frac{\omega_{c}}{T_{c}^{\prime}}$ in the case $\omega_{0}=5 \mathrm{TeV}$. Solid (and smooth) contour: $S^{\prime}=1$; dashed contour: $S^{\prime}=2$; dotted contour: $S^{\prime}=3$; dotted-dashed contour: $S^{\prime}=4$; uneven contour: $S^{\prime} \rightarrow \infty$. The mass ranges of $m_{H_{1}^{0}}$ and $m_{H_{2}^{ \pm}}$for the first-order phase transition are $0<m_{H_{1}^{0}}<1500 \mathrm{GeV}$ and $0^{2}<m_{H_{2}^{ \pm}}<3590 \mathrm{GeV}$, respectively 
Table 2 The mass ranges of $H_{1}^{0}$ and $H_{2}^{ \pm}$for the EWPT $S U(3) \rightarrow S U(2)$ to be the first-order phase transition, and their upper bounds as required by the condition $m_{\text {boson }}<2.2 \times T_{c}^{\prime}$

\begin{tabular}{llllr}
\hline$\omega(\mathrm{TeV})$ & $T_{c}^{\prime}(\mathrm{GeV})$ & $m_{H_{1}^{0}}(\mathrm{GeV})$ & $m_{H_{2}^{ \pm}}(\mathrm{GeV})$ & Upper bound $(\mathrm{GeV})$ \\
\hline 1 & 350 & $0<m_{H_{1}^{0}}<300$ & $0<m_{H_{2}^{ \pm}}<720$ & 770 \\
2 & 650 & $0<m_{H_{1}^{0}}<600$ & $0<m_{H_{2}^{ \pm}}<1440$ & 1430 \\
3 & 950 & $0<m_{H_{1}^{0}}<900$ & $0<m_{H_{2}^{ \pm}}<2150$ & 2090 \\
4 & 1300 & $0<m_{H_{1}^{0}}<1200$ & $0<m_{H_{2}^{ \pm}}<2870$ & 2860 \\
5 & 1600 & $0<m_{H_{1}^{0}}<1500$ & $0<m_{H_{2}^{ \pm}}<3590$ & 3520 \\
\hline
\end{tabular}

$V_{\mathrm{eff}}(v)=D\left(T^{2}-T_{0}^{2}\right) v^{2}-E T|v|^{3}+\frac{\lambda_{T}}{4} v^{4}$,

in which

$$
\begin{aligned}
& D=\frac{1}{24 v_{0}^{2}}\left[6 m_{W}^{2}\left(v_{0}\right)+6 m_{Y}^{2}\left(v_{0}\right)+3 m_{Z_{1}}^{2}\left(v_{0}\right)+m_{H^{0}}\left(v_{0}\right)\right. \\
& \left.+m_{H_{1}^{0}}\left(v_{0}\right)+2 m_{H_{2}^{+}}^{2}\left(v_{0}\right)+6 m_{t}^{2}\left(v_{0}\right)\right] \text {, } \\
& T_{0}^{2}=\frac{1}{D}\left\{\frac{m_{H}^{2}\left(v_{0}\right)+m_{H_{1}^{0}}\left(v_{0}\right)}{4}-\frac{1}{32 \pi^{2} v_{0}^{2}}\right. \\
& \times\left(6 m_{W}^{4}\left(v_{0}\right)+6 m_{Y}^{4}\left(v_{0}\right)+3 m_{Z_{1}}^{4}\left(v_{0}\right)\right. \\
& \left.\left.+m_{H^{0}}^{4}\left(v_{0}\right)+m_{H_{1}^{0}}^{4}\left(v_{0}\right)+2 m_{H_{2}^{+}}^{4}\left(v_{0}\right)-12 m_{t}^{4}\left(v_{0}\right)\right)\right\}, \\
& E=\frac{1}{12 \pi v_{0}^{3}}\left(6 m_{W}^{2}\left(v_{0}\right)+6 m_{Y}^{3}\left(v_{0}\right)+3 m_{Z_{1}}^{3}\left(v_{0}\right)\right. \\
& \left.+m_{H^{0}}^{3}\left(v_{0}\right)+m_{H_{1}^{0}}^{3}\left(v_{0}\right)+2 m_{H_{2}^{+}}^{3}\left(v_{0}\right)\right), \\
& \lambda_{T}=\frac{m_{H^{0}}^{2}\left(v_{0}\right)+m_{H_{1}^{0}}^{2}\left(v_{0}\right)}{2 v_{0}^{2}}\left\{1-\frac{1}{8 \pi^{2} v_{0}^{2}\left(m_{H^{0}}^{2}\left(v_{0}\right)+m_{H_{1}^{0}}^{2}\left(v_{0}\right)\right)}\right. \\
& \times\left[6 m_{W}^{4}\left(v_{0}\right) \ln \frac{m_{W}^{2}\left(v_{0}\right)}{b T^{2}}+6 m_{Y}^{4}\left(v_{0}\right) \ln \frac{m_{Y}^{2}\left(v_{0}\right)}{b T^{2}}\right. \\
& +3 m_{Z_{1}}^{4}\left(v_{0}\right) \ln \frac{m_{Z_{1}}^{2}\left(v_{0}\right)}{b T^{2}}+m_{H^{0}}^{4}\left(v_{0}\right) \ln \frac{m_{H^{0}}^{2}\left(v_{0}\right)}{b T^{2}} \\
& \left.\left.+2 m_{H_{2}^{+}}^{4}\left(v_{0}\right) \ln \frac{m_{H_{2}^{+}}^{2}\left(v_{0}\right)}{b T^{2}}-12 m_{t}^{4}\left(v_{0}\right) \ln \frac{m_{t}^{2}\left(v_{0}\right)}{b_{F} T^{2}}\right]\right\} \text {, }
\end{aligned}
$$

where $v_{0}$ is the value at which the zero-temperature effective potential $V_{\mathrm{eff}}^{0^{o} K}(v)$ gets the minimum. Here, we obtain $V_{\text {eff }}^{0^{o} K}(v)$ from $V_{\text {eff }}(v)$ in Eq. (45) by neglecting all terms in the form $F_{\mp}\left(\frac{m}{T}\right)$.

From the minimum conditions for $V_{\mathrm{eff}}^{0^{o} K}(v)$

$$
\begin{aligned}
& V_{\mathrm{eff}}^{0^{o} K}\left(v_{0}\right)=0,\left.\frac{\partial V_{\mathrm{eff}}^{0^{o} K}(v)}{\partial v}\right|_{v=v_{0}}=0 \\
& \left.\frac{\partial^{2} V_{\mathrm{eff}}^{0^{o} K}(v)}{\partial v^{2}}\right|_{v=v_{0}}=\left.\left[m_{H^{0}}^{2}(v)+m_{H_{1}^{0}}^{2}(v)\right]\right|_{v=v_{0}},
\end{aligned}
$$

we can see that in this EWPT, $m_{H^{0}}^{2}(v)$ and $m_{H_{1}^{0}}^{2}(v)$ generate the masses of the SM particles and the last mass part of $Y^{ \pm}$. We also have the minima of the effective potential (65):

$v=0, \quad v \equiv v_{c}=\frac{2 E T_{c}}{\lambda_{T_{c}}}$,

where $v_{c}$ is the critical VEV of $\phi$ at the broken state, and $T_{c}$ is the critical temperature of the phase transition, which is given by

$T_{c}=\frac{T_{0}}{\sqrt{1-E^{2} / D \lambda_{T_{c}}}}$.

We investigate the phase-transition strength

$S=\frac{v_{c}}{T_{c}}=\frac{2 E}{\lambda_{T_{c}}}$

of this EWPT. In the limit $E \rightarrow 0$, the transition strength $S \rightarrow 0$ and the phase transition is a second-order. To have a first-order phase transition, we requires $S \geq 1$. We plot $S$ as a function of $m_{H_{1}^{0}}\left(v_{0}\right)$ and $m_{H_{2}^{ \pm}}\left(v_{0}\right)$. As shown in Fig. 6, for the masses of $H_{2}^{ \pm}$and $H_{1}^{0}$, which are, respectively, in the ranges $250 \mathrm{GeV}<m_{H_{2}^{ \pm}(v)}<1200 \mathrm{GeV}$ and $0 \mathrm{GeV}<$ $m_{H_{1}^{0}(v)}<620 \mathrm{GeV}$, the transition strength is in the range $1 \leq S<3$.

Considering the requirement for the high-temperature expansion to be applicable on the effective potential (45), $\frac{m_{\text {boson }}}{T}<2.2$ [47], we show in Fig. 7 that with $T=T_{c} \sim$ $130 \mathrm{GeV}$, the mass ranges of $H_{2}^{ \pm}$and $H_{1}^{0}$ are, respectively, narrowed to

$255 \mathrm{GeV}<m_{H_{2}^{ \pm}}<280 \mathrm{GeV}$,

and

$0 \mathrm{GeV}<m_{H_{1}^{0}}<58 \mathrm{GeV}$

Corresponding with these ranges of mass, the range of phase-transition strength is narrowed to $1 \leq S<1.15$. Thus the EWPT $S U(2) \rightarrow U(1)$ is the first-order phase transition, but it seems quite weak. 


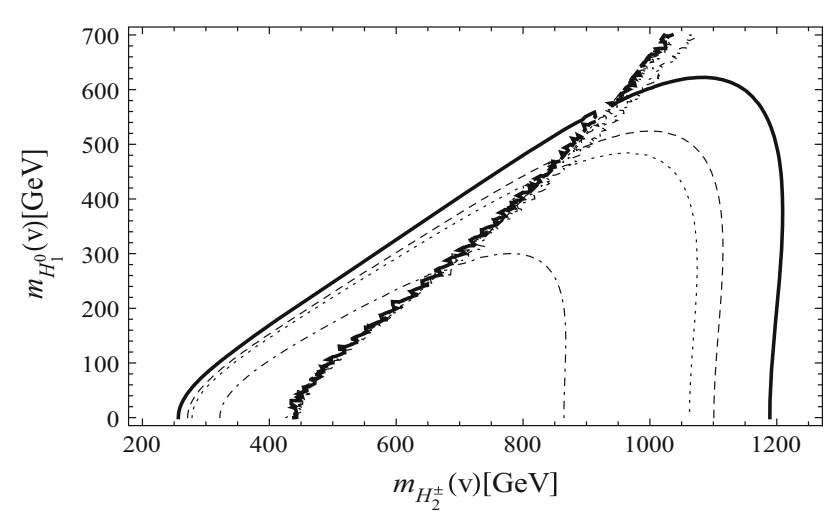

Fig. 6 The contours of transition strength $S=\frac{2 E}{\lambda T_{c}}$. Solid smooth contour: $S=1$; dashed smooth contour: $S=1.1$; dotted smooth contour: $S=1.15$; dash-dotted smooth contour: $S=1.5$; even contours: $S \rightarrow \infty$. The mass ranges of $m_{H_{1}^{0}}$ and $m_{H_{2}^{ \pm}}$for the EWPT $S U(2) \rightarrow U(1)$ to be the first-order are $0 \mathrm{GeV}<m_{H_{1}^{0}(v)}<620 \mathrm{GeV}$ and $250 \mathrm{GeV}<m_{H_{2}^{ \pm}(v)}<1200 \mathrm{GeV}$, respectively

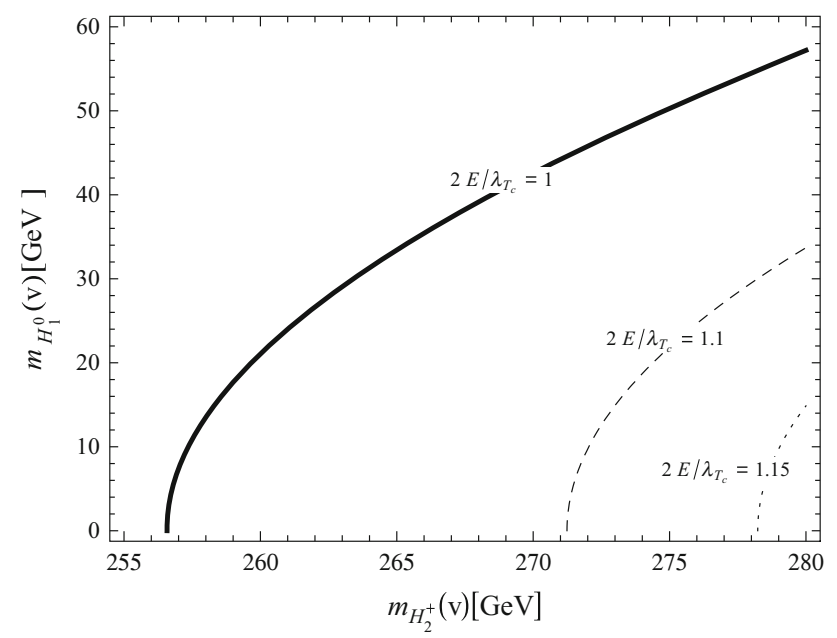

Fig. 7 The condition $\frac{m_{\text {boson }}}{T}<2.2$ narrows the mass ranges of $H_{2}^{ \pm}$and $H_{1}^{0}$ as well as the range of transition strength

As we can see in Eqs. (70) and (66), the new bosons contribute to the phase-transition strength $S$ via the parameters $E$ and $\lambda_{T_{c}}$. Hence these new bosons can be triggers for the EWPT $S U(2) \rightarrow U(1)$ to be the first-order.

In Fig. 8, we illustrate the dependence of the effective potential $V_{\text {eff }}(v)$ on the temperature. When the Universe cools through the phase-transition critical temperature $T_{c}$, the Higgs field $v$ tends to get a non-zero VEV $v_{0}$ which is in the range $0<v_{0}<246 \mathrm{GeV}$, and the second minimum of $V_{\text {eff }}(v)$ gradually appears at $v_{0}$. As the temperature drops from $T_{c}$, the second minimum becomes lower and the first minimum gradually disappears, while the VEV $v_{0}$ tends to $246 \mathrm{GeV}$. The tendency of $v_{0}$ can be seen in Fig. 9 where we show that $v_{0}$ reaches $246 \mathrm{GeV}$ for the temperatures which are

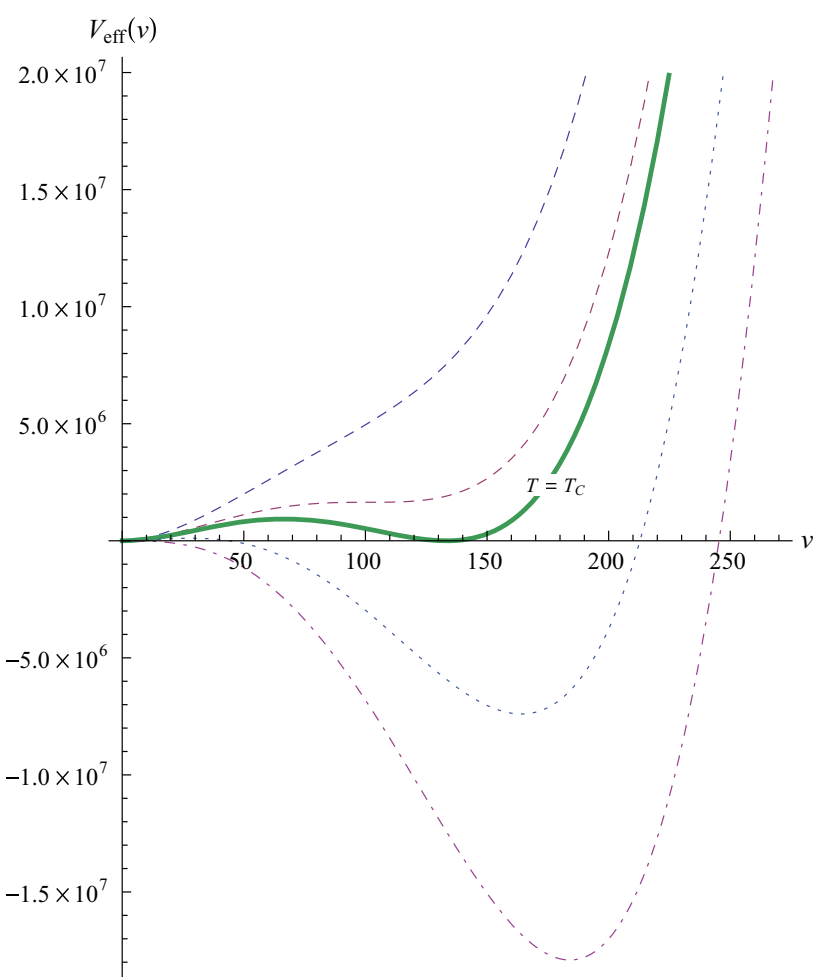

Fig. 8 The dependence of the effective potential $V_{\text {eff }}(v)$ on the temperature. With $m_{H_{1}^{0}}(v)=50 \mathrm{GeV}$ and $m_{H_{2}^{ \pm}}(v)=280 \mathrm{GeV}$, we have the critical temperature $T_{c}=127.974 \mathrm{GeV}$ and the phase-transition strength $S=1.03$. Solid line: $T_{c}$; lines above the solid line: $T>T_{c}$; lines under the solid line: $T<T_{c}$

far below $T_{c}$. At $0^{\circ} \mathrm{K}$, the non-zero minimum locates exactly at $v_{0}=246 \mathrm{GeV}$. This result is consistent with the SM.

\subsection{Constraint on the mass of the charged Higgs boson}

From the EWPT $S U(2) \rightarrow U(1)$, we have derived the mass ranges of $H_{2}^{+}(v)$ and $H_{1}^{0}(v)$ in Eqs. (71) and (72). So we have

$0 \mathrm{GeV}<m_{H_{1}^{0}}=\sqrt{m_{H_{1}^{0}}^{2}(v)+m_{H_{1}^{0}}^{2}(\omega)}<1501.12 \mathrm{GeV}$,

and we obtain

$2.149<\lambda_{4}<2.591$

and

$0<\frac{\lambda_{3}^{2}}{2 \lambda_{1}}<0.0556$,

From the phase transition $S U(3) \rightarrow S U(2)$, we have also derived

$0<\lambda_{4}<10.3$ 


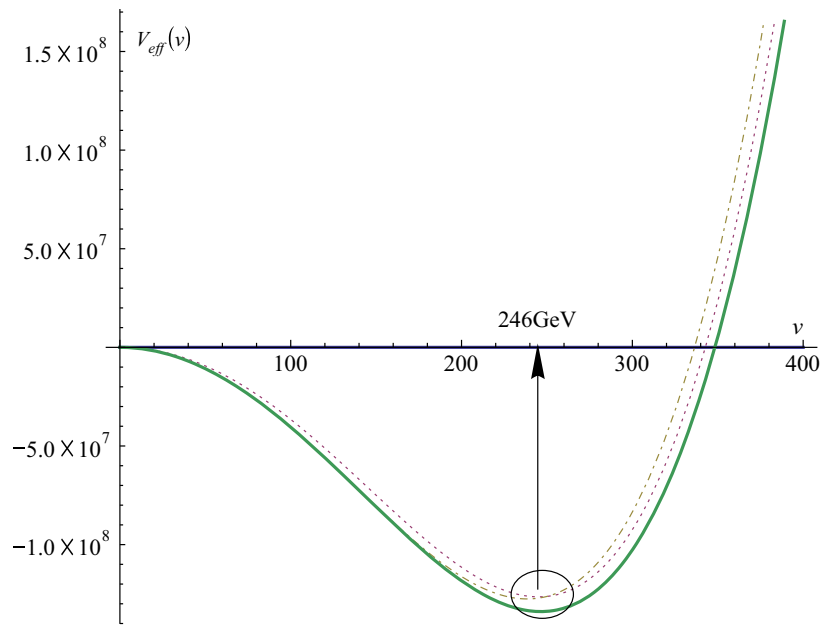

Fig. 9 The tendency of non-zero minimum for lower temperatures. We choose $m_{H_{1}^{0}}(v)=50 \mathrm{GeV}, m_{H_{2}^{ \pm}}(v)=280 \mathrm{GeV}$. Dotted-dashed line: $T=50 \mathrm{GeV}$. Dotted line: $T=10 \mathrm{GeV}$. Solid line: $T=1 \mathrm{GeV} . v_{0}$ reaches $246 \mathrm{GeV}$ as the temperature decreases

and

$0<\lambda_{1}<0.45$,

for any $\omega$. Eqs. (74)-(77) lead to $2.149<\lambda_{4}<2.591$; $0<\lambda_{1}<0.45$ and $0<\frac{\lambda_{3}^{2}}{2 \lambda_{1}}<0.0556$.

\section{Conclusion and outlooks}

We have investigated the EWPT in the E331 model using the high-temperature effective potential. Although the effective potential in the model depends complicatedly on three VEVs, $u, \omega$, and $v$, it can be transformed to a sum of two parts so that each part depends only on $\omega$ or $v$, which corresponds a stage of SSB. Therefore, the EWPT can be seen as a sequence of two EWPTs. The first, $S U(3) \rightarrow S U(2)$, takes place at the energy scale $\omega_{0}$ to generate the masses for the exotic quarks, the heavy gauge bosons $X^{0}$ and $Z_{2}$, as well as a mass part of $Y^{ \pm}$. The second, $S U(2) \rightarrow U(1)$, occurs at the scale $v_{0}$ to give the masses for the SM particles and the remaining mass part of $Y^{ \pm}$.

At the TeV scale, the EWPT $S U(3) \rightarrow S U(2)$ is strengthened by the new bosons and the exotic quarks to be the strongly first-order; if the masses of these new particles are about $10^{2}-10^{3} \mathrm{GeV}$, the phase-transition strength is in the range $1-13$. As the energy is lowered to the scale of $10^{2} \mathrm{GeV}$, the EWPT $S U(2) \rightarrow S U(1)$ is strengthened by only the new bosons; with the contributions of the mass parts from $H_{1}^{0}, H_{2}^{ \pm}$, and $Y^{ \pm}$, which are in the ranges $10-10^{2} \mathrm{GeV}$, the strength of this transition is about $1-1.15$. Therefore, both EWPTs can be the first-order; the $S U(3) \rightarrow S U(2)$ appears very strong, while the $S U(2) \rightarrow S U(1)$ seems quite weak.
However, both of these first-order EWPTs can be sufficiently strong to provide B violation necessary for baryogenesis, as shown via the parameter ranges which we have specified. If $H_{1}^{0}$ and $H_{2}^{ \pm}$exist, their contributions to the strengths of each EWPT are meaningly large. In this case, the sequence of strongly first-order EWPTs in the model may provide a source of large deviations from thermal equilibrium. The model may fully describe the continual existence of BAU since it was generated in the early Universe.

In subsequent work, we will investigate the electroweak sphalerons as well as the $\mathrm{C}$ - and $\mathrm{CP}$ - violating interactions to know if the model possesses all necessary components for EWBG.

Acknowledgments This research is funded by Vietnam National Foundation for Science and Technology Development (NAFOSTED) under grant number 103.01-2014.51.

Open Access This article is distributed under the terms of the Creative Commons Attribution 4.0 International License (http://creativecomm ons.org/licenses/by/4.0/), which permits unrestricted use, distribution, and reproduction in any medium, provided you give appropriate credit to the original author(s) and the source, provide a link to the Creative Commons license, and indicate if changes were made. Funded by SCOAP ${ }^{3}$.

\section{References}

1. A.D. Sakharov, JETP Lett. 5, 24 (1967)

2. V. Mukhanov, Physical Foundations, of Cosmology, Michael Dine, Robert G. Leigh, Patrick Huet, Andrei Linde, and Dmitri Linde, Phys. Rev. D. 46, 550 (1992). (Cambridge University Press, Cambridge 2005)

3. A.G. Cohen, D.B. Kaplan, A.E. Nelson, Ann. Rev. Nucl. Part. Sci. 43, 27 (1993)

4. A.G. Cohen, in Physics at the Frontiers of the Standard Model, 2nd Rencontres du Vietnam (Ho Chi Minh City, 1995), pp. 311315

5. K. Kajantie, M. Laine, K. Rummukainen, M. Shaposhnikov, Phys. Rev. Lett. 77, 2887 (1996)

6. F. Csikor, Z. Fodor, J. Heitger, Phys. Rev. Lett. 82, 21 (1999)

7. J. Grant, M. Hindmarsh, Phys. Rev. D. 64, 016002 (2001)

8. M. D'Onofrio, K. Rummukainen, A. Tranberg, JHEP 08, 123 (2012)

9. M. Bastero-Gil, C. Hugonie, S.F. King, D.P. Roy, S. Vempati, Phys. Lett. B 489, 359 (2000)

10. A. Menon, D.E. Morrissey, C.E.M. Wagner, Phys. Rev. D 70, 035005 (2004)

11. S.W. Ham, S.K. Oh, C.M. Kim, E.J. Yoo, D. Son, Phys. Rev. D 70, 075001 (2004)

12. J.R. Espinosa, T. Konstandin, F. Riva, Nucl. Phys. B 854, 592 (2012)

13. S. Kanemura, E. Senaha, T. Shindou, T. Yamada, JHEP 1305, 066 (2013)

14. D.J.H. Chung, A.J. Long, Phys. Rev. D 81, 123531 (2010)

15. J.M. Cline, G. Laporte, H. Yamashita, S. Kraml, JHEP 0907, 040 (2009)

16. S. Kanemura, Y. Okada, E. Senaha, Phys. Lett. B 606, 361-366 (2005)

17. S.W. Ham, S.-A. Shim, S.K. Oh, Phys. Rev. D 81, 055015 (2010)

18. G. Barenboim, N. Rius, Phys. Rev. D 58, 065010 (1998) 
19. S. Das, P.J. Fox, A. Kumar, N. Weiner, JHEP 1011, 108 (2010)

20. D. Chung, A.J. Long, Phys. Rev. D. 84, 103513 (2011)

21. M. Carena, N.R. Shaha, C.E.M. Wagner, Phys. Rev. D 85, 036003 (2012)

22. A. Ahriche, S. Nasri, Phys. Rev. D 85, 093007 (2012)

23. D. Borah, J.M. Cline, Phys. Rev. D 86, 055001 (2013)

24. F. Pisano, V. Pleitez, Phys. Rev. D 46, 410 (1992)

25. P.H. Frampton, Phys. Rev. Lett. 69, 2889 (1992)

26. R. Foot et al., Phys. Rev. D 47, 4158 (1993)

27. M. Singer, J.W.F. Valle, J. Schechter, Phys. Rev. D 22, 738 (1980)

28. R. Foot, H.N. Long, T.A. Tran, Phys. Rev. D 50 R34 (1994). arXiv:hep-ph/9402243

29. J.C. Montero, F. Pisano, V. Pleitez, Phys. Rev. D 47, 2918 (1993)

30. H.N. Long, Phys. Rev. D 53, 437 (1996)

31. H.N. Long, Phys. Rev. D 54, 4691 (1996)

32. H.N. Long, Mod. Phys. Lett. A 13, 1865 (1998)

33. C.A.deS. Pires, O.P. Ravinez, Phys. Rev. D 58, 035008 (1998)

34. A. Doff, F. Pisano, Mod. Phys. Lett. A 14, 1133 (1999)

35. A. Doff, F. Pisano, Phys. Rev. D 63, 097903 (2001)
36. P.V. Dong, H.N. Long, Int. J. Mod. Phys. A 21, 6677 (2006)

37. V.Q. Phong, V.T. Van, H.N. Long, Phys. Rev. D 88, 096009 (2013)

38. W.A. Ponce, Y. Giraldo, L.A. Sanchez, Phys. Rev. D 67, 075001 (2003)

39. P.V. Dong, H.N. Long, D.T. Nhung, D.V. Soa, Phys. Rev. D 73, 035004 (2006)

40. P.V. Dong, H.N. Long, Adv. High Energy Phys. 2008, 739492 (2008)

41. P.V. Dong, Tr.T. Huong, D.T. Huong, H.N. Long, Phys. Rev. D 74, 053003 (2006)

42. P.V. Dong, H.N. Long, D.V. Soa, Phys. Rev. D 73, 075005 (2006)

43. P.V. Dong, H.N. Long, D.V. Soa, Phys. Rev. D 75, 073006 (2007)

44. P.V. Dong, H.T. Hung, H.N. Long, Phys. Rev. D 86, 033002 (2012)

45. Particle Data Group: J. Beringer et al., Phys. Rev. D 86, 010001 (2012)

46. A.G. Dias, V. Pleitez, Phys. Rev. D 80, 056007 (2009)

47. Greg W. Anderson, Lawrence J. Hall, Phys. Rev. D 45, 2685-2698 (1992) 